\title{
Thrombotic occlusion of the ostial left main coronary artery in a patient with acute coronary syndrome
}

\author{
E. Tatli
}

Ostial left main coronary artery (LMCA) occlusion is rarely seen in patients with acute coronary syndrome. Acute coronary syndrome resulting from an LMCA occlusion is associated with a significant morbidity and mortality rate, if it is managed with fibrinolysis. Electrocardiography can predict LMCA occlusion in patients with acute coronary syndrome. We report a 52-year-old male who presented with acute coronary syndrome and ostial LMCA occlusion. (Neth Heart J 2009;17: 295-6.)

Keywords: ostial left main occlusion, acute coronary syndrome, electrocardiography

A 52-year-old male presented with acute coronary syndrome and cardiogenic shock. The patient had no history of previous illnesses or admissions to hospital, specifically he had never received radiotherapy. He was not on any medications and did not abuse illicit substances. He had no history of hypertension, smoking, hypercholesterolaemia, or diabetes. He did not have a family history of premature coronary artery disease. Electrocardiography (ECG) on admission showed ST elevation in lead aVR and VI (figure 1). Emergent coronary angiogram obtained immediately after placing an intra-aortic balloon pump revealed ostial thrombotic occlusion in the left main coronary artery (LMCA, figure 2 ). We performed direct coronary angioplasty intervention with the aid of tirofiban (Aggrastat) infusion (figure 3). No residual thrombus and stenosis remained after high pressure balloon dilatation with $3.0 \times 15$ balloon at $16 \mathrm{~atm}$, for 30 seconds (figure 4 ). Inotropic support was maintained with dopamine and intra-aortic balloon pump in

\section{E. Tatli}

Department of Cardiology, Trakya University, Edirne, Turkey

Correspondence to: E. Tatli

Department of Cardiology, School of Medicine, Trakya

University, Edirne, Turkey

E-mail: ersantatli@yahoo.com the coronary care unit. The patient was asymptomatic one week after the procedure.

\section{Discussion}

Electrocardiography is an appropriate bedside tool used in the emergency department to make a rapid diagnosis of acute coronary syndrome, allowing physicians to select appropriate therapy and to predict potential cardiovascular complications. There is no general agreement on the typical ECG pattern of sudden occlusion of the LMCA. Many different ECG patterns have been described by authors, either as case reports or as small series. ${ }^{1-4}$

Hori et al. described 13 patients with acute myocardial infarction (MI) caused by total occlusion of the LMCA during a period of eight years. ${ }^{1}$ ST elevation in lead aVR was present in $69 \%$ of patients, and five of the six non-survivors had ST-segment elevation in lead aVR as well as aVL. In a descriptive study of 16 patients by Yamaji et al. ${ }^{2}$ ST-segment elevation in lead aVR with less elevation in lead Vl proved to be an important predictor of LMCA occlusion in patients admitted within 12 hours of the onset of acute MI, as in our patient. Other ECG patterns caused by LMCA occlusion are anterior ST-segment elevation, ST-segment depression in the precordial leads, right bundle branch block, and ST-segment elevation in leads I and aVL. ${ }^{3}$

Acute LMCA occlusion is a serious clinical condition. Despite its low incidence, the prognosis is often poor. Thus, it is important to be able to identify patients with LMCA occlusion and acute coronary syndrome. Electrocardiographic patterns supporting the occlusion of LMCA involve: ${ }^{4}$

1. ST-segment elevation in both aVR and aVL (highest specificity 98\%)

2. ST elevation in aVR less than the ST elevation in VI

3. Right bundle branch block and left anterior fascicular block

4. ST depression in leads II, III or aVF (highest sensitivity $88 \%$ )

In conclusion, acute LMCA occlusion is associated with a markedly higher mortality rate and may be more amenable to mechanical reperfusion strategies rather 


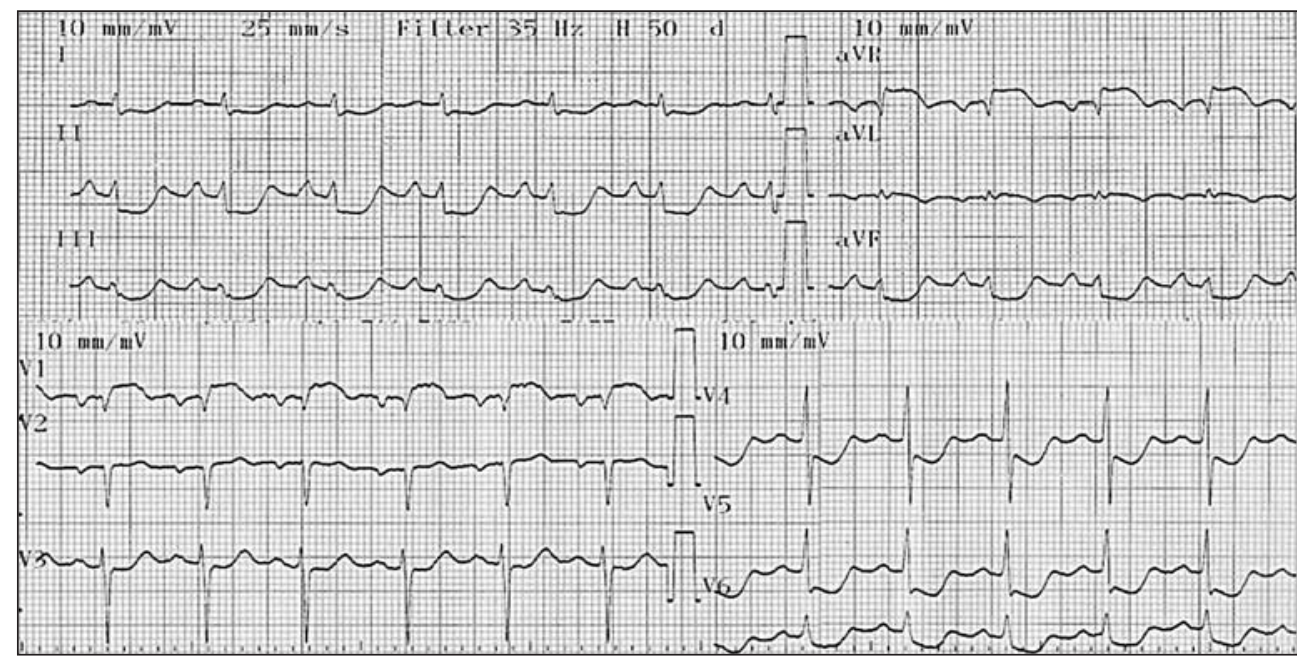

Figure 1. ECG on admission showing ST elevation in lead aVR and VI and $S T$ depression in lead D1, D2, D3, aVF and V3-V6.

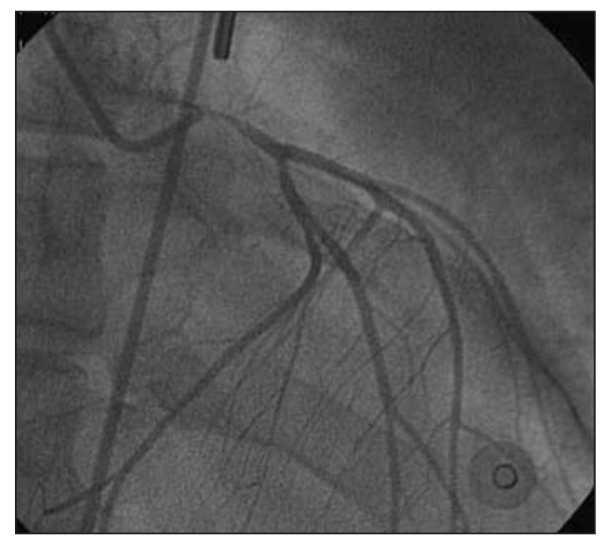

Figure 2. Coronary angiogram showing $90 \%$ thrombotic occlusion of the ostial LMCA.

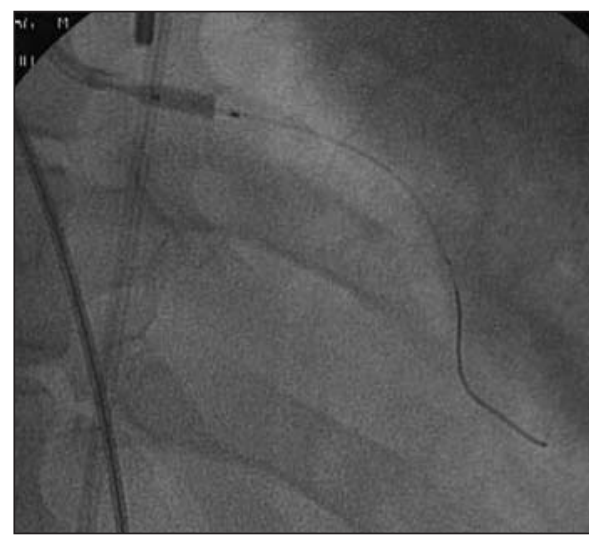

Figure 3. Coronary angiogram showing balloon dilatation.

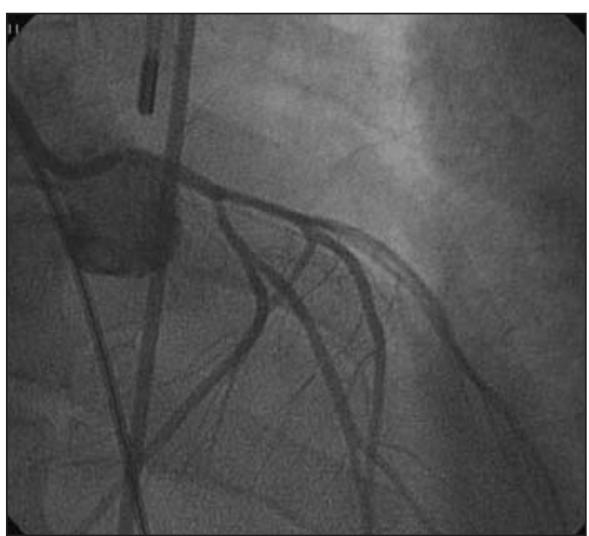

Figure 4. Coronary angiogram after balloon dilatation showing improved LMCA flow without residual stenosis. than fibrinolysis. Early recognition and faster percutaneous coronary intervention may decrease the mortality rate. Thus, it is important to know the electrocardiographic patterns which can predict LMCA occlusion.

\section{References}

1 Hori T, Kurosawa T, Yoshida M, Yamazoe M, Aizawa Y, Izumi T. Factors predicting mortality in patients after myocardial infarction caused by left main coronary artery occlusion: significance of ST segment elevation in both aVR and aVL leads. Jpn Heart J 2000; 41:571.
2 Yamaji H, Iwasaki K, Kusachi S, Murakami T, Hirami R Hamamoto $\mathrm{H}$, et al. Prediction of acute left main coronary artery obstruction by 12-lead electrocardiography. ST segment elevation in lead aVR with less ST segment elevation in lead V1. J Am Coll Cardiol 2001;38:1348-54.

3 Jong GP, Ma T, Chou P, Shyu MY, Tseng WK, Chang TC. Reciprocal changes in 12-lead electrocardiography can predict left main coronary artery lesion in patients with acute myocardial infarction. Int Heart J 2006;47:13-20.

4 Bhatia V, Parida AK, Pandey AK, Kaul U. 12 lead ECG in the detection of left main coronary artery occlusion during acute coronary syndromes. Int J Cardiol 2007;116:e83-4. 